\title{
Nuclear $\beta$ catenin expression is related to unfavourable outcome in oropharyngeal and hypopharyngeal squamous cell carcinoma
}

M J Pukkila, J A Virtaniemi, E J Kumpulainen, R T Pirinen, R T Johansson, H J Valtonen, M T Juhola, V-M Kosma

Abstract

Aims-To investigate the expression of $\alpha$,

Department of Otorhinolaryngology, University of Kuopio, Kuopio University Hospital, PO Box 1777, FIN-70211, Kuopio,

Finland

M J Pukkila

J A Virtaniemi

Department of Oncology, University

of Kuopio, Kuopio

University Hospital

$\mathrm{R} \mathrm{T}$ Johansson

E J Kumpulainen

Department of Pathology and Forensic Medicine, University of Kuopio, Kuopio University Hospital, PO Box 1627, FIN-70211 Kuopio,

Finland

V-M Kosma

R T Pirinen

Department of Pathology, Central Hospital of Jyväskylä, FIN-40620, Jyväskylä,

Finland

M T Juhola

Department of Otorhinolaryngology, Central Hospital of Jyväskylä

H J Valtonen

Correspondence to: Acting Professor Kosma VeliMatti.Kosma@uku.fi

Accepted for publication 25 May 2000

Table 1 Studies on catenin expression in cancers of the head and neck

\begin{tabular}{|c|c|c|c|c|c|}
\hline Authors & $\begin{array}{l}\text { Patient } \\
\text { number }\end{array}$ & Location & $\begin{array}{l}\text { Catenins } \\
\text { studied }\end{array}$ & Method & $\begin{array}{l}\text { Association with } \\
\text { survival }\end{array}$ \\
\hline Andrews et al $(1997)^{14}$ & 51 & $\begin{array}{l}M(10) \\
\text { OP (5) } \\
\mathrm{L}(6) \\
\mathrm{HP}(10) \\
\text { Other (5) }\end{array}$ & $\alpha, \beta$ & IHC & None \\
\hline Williams et al $(1998)^{15}$ & 12 & M & $\alpha, \beta, \gamma$ & IHC & Not studied \\
\hline Bagutti et al $(1998)^{16}$ & 22 & M & $\alpha, \beta, \gamma$ & IHC, IF & Not studied \\
\hline Hirvikoski et al $(1998)^{17}$ & 159 & $\mathrm{~L}$ & $\alpha$ & IHC & Yes, suggestive \\
\hline Zheng et al (1999) ${ }^{18}$ & 74 & NP & $\beta$ & IHC & Yes \\
\hline Lo Muzio et al (1999) 19 & 30 & $\mathrm{M}$ & $\beta, \gamma$ & IHC & Not studied \\
\hline Lou et al (1999) $)^{20}$ & 42 & NP & $\alpha, \beta, \gamma$ & IHC, IB & Not studied \\
\hline Our series & 116 & $\begin{array}{l}\mathrm{OP}(88) \\
\mathrm{HP}(50)\end{array}$ & $\alpha, \beta, \gamma$ & IHC & Yes (nuclear $\beta$ ) \\
\hline
\end{tabular}

IB, immunoblotting; IF, immunofluorescence; IHC, immunohistochemistry; HP, hypopharynx; $\mathrm{L}$, larynx; M, mouth; NP, nasopharynx; OP, oropharynx.
Among malignant tumours in the head and neck region, pharyngeal cancer has the worst prognosis. ${ }^{1}$ Histologically, most of the tumours are squamous cell carcinomas, whereas mucoepidermoid carcinomas, lymphomas, and other tumour types are less frequent. Approximately 750 new cases of pharyngeal cancer are diagnosed annually in Scotland and England, whereas in Finland the corresponding number is about 80.

Cadherins are integral transmembrane glycoproteins responsible for calcium dependent intercellular adhesion. The normal function of $\mathrm{E}$ cadherin depends on binding to the cytoplasmic anchoring proteins, namely: $\alpha$ catenin $(102 \mathrm{kDa}), \beta$ catenin $(88 \mathrm{kDa})$, and $\gamma$ catenin $(80 \mathrm{kDa}){ }^{2}$ Both $\beta$ and $\gamma$ catenins bind directly to the cytoplasmic part of the $\mathrm{E}$ cadherin molecule, whereas $\alpha$ catenin connects the cadherin bound $\beta$ or $\gamma$ catenins to the actin microfilament network of the cellular cytoskeleton. ${ }^{34}$ In addition to intercellular adhesion, catenins also influence differentiation, maintenance of the architecture of differentiated tissues, polarity, migration, cell proliferation, and signalling transduction for gene transcription, thereby linking the cell surface to cytoplasmic and nuclear events. ${ }^{5}$ These interactions are mediated by the association of catenins not only with $\mathrm{E}$ cadherin, but also with other transmembrane, cytoplasmic, and nuclear target proteins. ${ }^{5-7}$

The immunohistochemical detection of catenins may reflect intercellular adhesion more directly than that of $\mathrm{E}$ cadherin alone. ${ }^{8}$ An association between reduced catenin expression and unfavourable prognosis has been noted previously in many carcinomas. ${ }^{8-13}$ However, the role of different catenins in squamous cell carcinomas of the head and neck (SCCHN) is unclear (table 1). ${ }^{14-20}$ To elucidate further the relations between catenins and clinicopathological factors, as well as survival, we studied the expression of $\alpha, \beta$, and $\gamma$ catenins in a large cohort of primary pharyngeal squamous cell carcinomas (PSCCs).

\section{Methods}

PATIENTS AND CLINICAL DATA

Clinical data and the original haematoxylin and eosin (H\&E) stained sections from 161 patients with primary PSCC diagnosed between 1975 and 1998 in eastern Finland were identified from the hospital records and from 
the population based Finnish Cancer Registry. Tumours with a nasopharyngeal origin were excluded.

The clinical data from the 161 cases were reviewed by one oncologist (EK) and two otolaryngologists (MP and JV). The original histological sections of the tumours were reviewed by an experienced pathologist (V$\mathrm{MK})$. Of the 161 cases, 23 were excluded because of non-squamous histology $(n=11)$, carcinoma in situ histology $(n=2)$, location of the primary tumour in the oral cavity $(n=3)$ or the larynx $(n=3)$, or insufficient clinical data $(\mathrm{n}=4)$. After primary evaluation, 138 cases (105 men, 33 women) were studied. The tumours were staged according to the Union Internationale Contre le Cancer (UICC) classification (1997), ${ }^{21}$ based on written hospital records of clinical otolaryngological status, endoscopy, and chest $x$ ray. The performance status according to the Karnofsky scale at the time of diagnosis was coded..$^{22}$ All patients were regularly followed up by an otolaryngologist or oncologist until death or May 1999. None of the patients was lost from the follow up.

HISTOLOGICAL METHODS

When reviewing the original $\mathrm{H} \& \mathrm{E}$ stained sections, the histological differentiation of the primary tumour was re-evaluated and classified as good $(\mathrm{n}=34)$, moderate $(n=63)$, or poor $(n=41)$ by the same pathologist (V-MK) according to the World Health Organisation (WHO) classification. ${ }^{23}$ The most representative block was chosen, and cut into $5 \mu \mathrm{m}$ thick sections for immunohistochemical staining.

IMMUNOHISTOCHEMISTRY

Rehydrated paraffin wax embedded sections were washed twice in phosphate buffered saline (PBS) and heated in a microwave oven at $600 \mathrm{~W}$ for three cycles of five minutes each in $0.05 \mathrm{~mol} /$ litre Tris/ $\mathrm{HCl}$ buffer $(\mathrm{pH} 9.7$ ) for $\alpha$ catenin, and $0.05 \mathrm{~mol} /$ litre citrate buffer ( $\mathrm{pH}$ 9.7) for $\beta$ and $\gamma$ catenins. Endogenous peroxidase activity was blocked with $5 \% \mathrm{H}_{2} \mathrm{O}_{2}$ in distilled water for five minutes and washed three times for five minutes in distilled water and twice for five minutes in PBS. After treatment with $1.5 \%$ normal horse serum (Vectastain Elite ABC kit; Vector Laboratories, Burlingame, California, USA), mouse anti- $\alpha$, anti- $\beta$, or anti- $\gamma$ catenin monoclonal antibodies (Transduction Laboratories, Lexington, Kentucky, USA) were applied to the sections at a dilution of $1 / 200,1 / 1000$, and $1 / 200$, respectively, in PBS with $1 \%$ bovine serum albumin and incubated for $24 \mathrm{~h}$ at $4^{\circ} \mathrm{C}$. Thereafter, the samples were washed and biotinylated secondary antibody and avidin-biotin peroxidase reagent (Vectastain Elite ABC kit; Vector Laboratories) were applied to detect bound primary antibody. Diaminobenzidine tetrahydrochloride (DAB; Sigma, St Louis, Missouri, USA) was used to demonstrate peroxidase activity. The slides were counterstained with Mayer's haematoxylin, dehydrated, cleared, and mounted with DePex (BDH, Poole, Dorset, UK). Normal epithelium and glandu- lar tissue served as internal positive controls. In addition, strongly positive tissue samples for each catenin from other series (lung and intestine) were used as positive controls in each staining batch. Samples from the same series without the use of primary antibody served as negative controls.

EVALUATION OF STAINING

The fraction of tumour cells in the sample with membranous staining for $\alpha, \beta$, and $\gamma$ catenins was primarily analysed on a continuous scale. For statistical analysis, all groups were divided into two subgroups using median percentages: tumours in which $\geqslant 90 \%$ of the cancer cells stained were considered normal, whereas those with reduced staining $(<90 \%)$ were considered abnormal. The same cut off point has been used successfully in previous studies investigating catenin expression in cancer. ${ }^{9}{ }^{10} \mathrm{In}$ tumours with abnormal catenin expression, discontinuous or absent membranous staining was seen, with or without cytoplasmic staining. Nuclear $\beta$ catenin staining was recorded as positive or negative. In positive samples, nuclear staining was seen in more than $10 \%$ of tumour cells. All slides were evaluated by three observers (V-MK, RP, and MP), who were unaware of the clinical and histopathological data. Disagreement in the assessment of staining was found in less than $10 \%$ of the slides examined. These samples were re-evaluated simultaneously by two observers (V-MK and MP) with a dual head microscope and the final staining pattern was settled. Necrotic tumour areas were ignored.

\section{STATISTICAL ANALYSIS}

SPSS for Windows Release 8.0 was used for statistical analysis. The representativeness of those patients with tissue samples available for immunohistochemistry $(n=116)$ of the whole patient group $(n=138)$ was checked with a $\chi^{2}$ goodness of fit test. The association between catenin staining patterns and clinicopathological variables was analysed with the $\chi^{2}$ test. The univariate analysis of overall survival was calculated with statistics presented by Gehan and was based on the life table method (log rank analysis). Cancer related overall survival (OS) was calculated from the date of primary diagnostic biopsy to the end of follow up or death. Only deaths from pharyngeal carcinoma were included as outcome events in the survival analysis. Multivariate overall survival analysis (Cox proportional hazards model) was performed in a stepwise manner. The enter limit was $p<0.05$ and the removal limit $p \geqslant 0.1$. Baseline covariates used in the Cox proportional hazard model were age, sex, site (oropharynx or hypopharynx), differentiation (good/moderate or poor), T class (categorical, reference class T1), presence of lymph node metastases (N0 or N+), M class, Karnofsky status $(\geqslant 70$ or $\leqslant 60)$, catenin expression (normal or reduced), and nuclear $\beta$ catenin staining (negative or positive). 
Table 2 Clinicopathological and treatment data of the whole patient group $(n=138)$

\begin{tabular}{|c|c|}
\hline Variable & Number of patients (\%) \\
\hline \multicolumn{2}{|l|}{$\overline{S e x}$} \\
\hline Male & $105(76)$ \\
\hline Female & $33(24)$ \\
\hline \multicolumn{2}{|l|}{ Site } \\
\hline Oropharynx & $88(64)$ \\
\hline Hypopharynx & $50(36)$ \\
\hline \multicolumn{2}{|l|}{ Tumour status } \\
\hline $\mathrm{T} 1$ & $23(17)$ \\
\hline $\mathrm{T} 2$ & $46(33)$ \\
\hline T3 & $25(18)$ \\
\hline $\mathrm{T} 4$ & $44(32)$ \\
\hline \multicolumn{2}{|l|}{ Nodal status } \\
\hline No & $81(59)$ \\
\hline N1 & $21(15)$ \\
\hline $\mathrm{N} 2$ & $32(23)$ \\
\hline N3 & $4(3)$ \\
\hline \multicolumn{2}{|l|}{ Distant metastases } \\
\hline M0 & $132(96)$ \\
\hline M1 & $6(4)$ \\
\hline \multicolumn{2}{|l|}{ Stage } \\
\hline I & $16(12)$ \\
\hline II & $28(20)$ \\
\hline III & $26(19)$ \\
\hline IV & $68(49)$ \\
\hline \multicolumn{2}{|l|}{ Histological differentiation } \\
\hline Good & $34(24)$ \\
\hline Moderate & $63(46)$ \\
\hline Poor & $41(30)$ \\
\hline \multicolumn{2}{|l|}{ Performance status (Karnofsky) } \\
\hline 90 & $8(6)$ \\
\hline 80 & $21(15)$ \\
\hline 70 & $62(45)$ \\
\hline 60 & $29(21)$ \\
\hline 50 & $15(11)$ \\
\hline 40 & $3(2)$ \\
\hline \multicolumn{2}{|l|}{ Primary treatment } \\
\hline Radiotherapy & $86(62)$ \\
\hline Surgery & $8(6)$ \\
\hline Surgery and radiotherapy & $38(28)$ \\
\hline Chemotherapy & $2(1)$ \\
\hline No treatment & $4(3)$ \\
\hline \multicolumn{2}{|l|}{ Goal of the primary treatment } \\
\hline Curative & $120(87)$ \\
\hline Palliative & $14(10)$ \\
\hline
\end{tabular}

ETHICS

The research plan was approved by the research ethical committee of Kuopio University and Kuopio University Hospital. Permission for obtaining data from the Finnish Cancer Registry and from hospital records was granted by the Finnish Ministry of Social Affairs and Health (permission No 88/08/97).

\section{Results}

CLINICAL DATA

Table 2 summarises the patient and treatment characteristics. The median age of the patients at the time of diagnosis was 64 (range, 36-89) years. The median follow up time was 43 (range, 1-332) months. The distributions of the baseline characteristics (sex, age, performance index, tumour differentiation, and stage) among the patients with tissue samples available for immunohistochemistry $(n=116)$ did not differ from the original patient group $(n=138)(p \geqslant 0.29)$. Forty five $(33 \%)$ patients remained free of disease during the follow up. A complete response was not achieved in $42(30 \%)$ patients, and in $51(37 \%)$ patients the cancer relapsed after a disease free period. At the end of follow up, 21 (15\%) patients were alive, $87(63 \%)$ patients had died as a result of PSCC, and $30(22 \%)$ patients had died from a cause not related to PSCC. Ten patients died of secondary malignant tumours. Radiotherapy alone was the most common
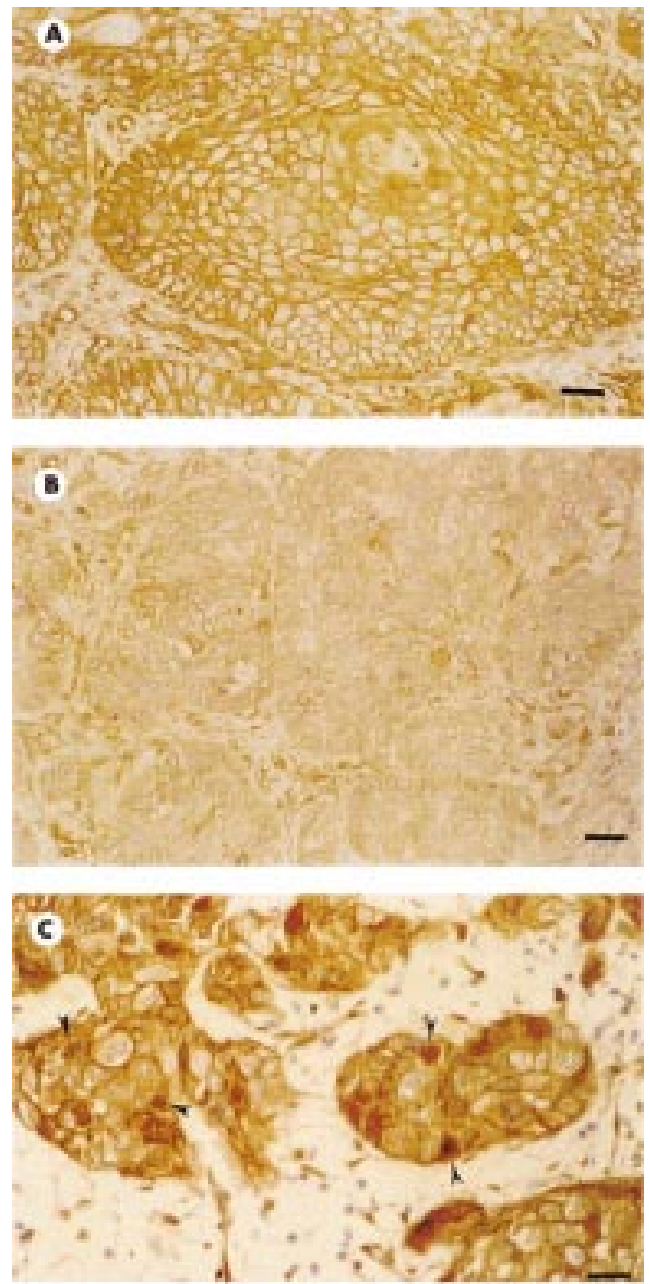

Figure 1 Immunohistochemical expression of $\beta$ catenin in human pharyngeal squamous cell carcinoma. (A) Normal expression on carcinoma cell membranes; bar, $50 \mu \mathrm{m}$. (B) Reduced expression; bar, $20 \mu m$. (C) Reduced expression, arrowheads indicate nuclear positivity; bar, $50 \mu \mathrm{m}$.

treatment modality. The primary treatment was considered curative in 120 and palliative in 14 patients. Four patients received no cancer treatment because of poor general condition.

CATENIN EXPRESSION AND ASSOCIATION WITH CLINICOPATHOLOGICAL CHARACTERISTICS The median percentage of positively stained cells for all three catenins was 90\% (range, $5-100 \%)$. Expression was reduced in $57(49 \%)$ tumours for $\alpha$ catenin, in $32(28 \%)$ tumours for $\beta$ catenin (fig $1 \mathrm{~A}$ and B), and in $30(26 \%)$ tumours for $\gamma$ catenin. Nuclear $\beta$ catenin expression (fig 1C) was positive in 27 (23\%) tumours.

Table 3 details the expression patterns (normal or reduced) of catenins. The membranous expression patterns of various catenins were significantly interrelated $(p \leqslant 0.025)$. Abnormal $\gamma$ catenin expression was associated significantly with tumour dedifferentiation $(p=0.04)$ (table 4). No association between other catenin expression patterns and recorded clinicopathological variables was detected.

CATENIN EXPRESSION AND SURVIVAL

In univariate analysis, nuclear $\beta$ catenin expression was associated with unfavourable 
Table 3 The expression pattern of catenins in our patient population $(n=116)$

\begin{tabular}{llll}
\hline \multicolumn{1}{c}{ Catenin } & & & \\
\cline { 1 - 2 }$a$ & $\beta$ & $\gamma$ & Number $(\%)$ \\
\hline Normal & Normal & Normal & $47(40)$ \\
Normal & Normal & Reduced & $6(5)$ \\
Normal & Reduced & Normal & $5(4)$ \\
Normal & Reduced & Reduced & $1(1)$ \\
Reduced & Normal & Normal & $20(17)$ \\
Reduced & Normal & Reduced & $11(10)$ \\
Reduced & Reduced & Normal & $14(12)$ \\
Reduced & Reduced & Reduced & $12(11)$ \\
\hline
\end{tabular}

Table 4 Association of reduced catenin expression with tumour differentiation $(n=116)$

\begin{tabular}{llll}
\hline & \multicolumn{2}{l}{$\begin{array}{l}\text { Number of tumours with reduced catenin } \\
\text { expression }\end{array}$} \\
\cline { 2 - 4 } Catenin \\
\cline { 2 - 4 } Differentiation & $\alpha$ & $\beta$ & $\gamma$ \\
\hline Good & 10 & 6 & 4 \\
Moderate & 28 & 13 & 11 \\
Poor & 19 & 13 & 15 \\
Total & 57 & 32 & 30 \\
$\begin{array}{l}\text { Association } \\
\chi^{2} \text { Value } \\
\text { p Value }\end{array}$ & 2.1 & 1.6 & 6.5 \\
\hline
\end{tabular}

$\star$ Differentiation in our study (116 tumours): $\operatorname{good}(\mathrm{n}=27)$ moderate $(n=52)$, poor $(n=37)$.

disease outcome $(p=0.001)$. The overall survival was also predicted by $\mathrm{T}$ class $(\mathrm{p}<0.00005)$, nodal status $(\mathrm{p}=0.03), \mathrm{M}$ class $(p=0.002)$, stage $(p<0.00005)$, and performance status $(\mathrm{p}<0.00005)$. Neither $\alpha$ nor $\gamma$ catenin was associated with survival. In multivariate analysis of overall survival, only nuclear $\beta$ catenin expression, $T$ class, and performance status remained significant (table 5).

\section{Discussion}

In the literature, both qualitative and quantitative changes in cadherins or catenins have been shown to be associated with dedifferentiation, dissemination of tumour cells from primary location, and prognosis in many human tumours, ${ }^{8-13}$ 24-26 including head and neck carcinomas. ${ }^{14} 161719$ Yet, the exact mechanisms behind changes in the expression of cadherin or catenin in cancer remain unclear. In SCCHN, the association of reduced E cadherin expression with dedifferentiation and metastasis has been demonstrated. ${ }^{27-30}$ However, the role of catenins in SCCHN has been investigated less extensively (table 1 ). ${ }^{14-20}$ Some reports have indicated that catenins are essential for the normal function of cadherin mediated adhesion and that their loss is reflected in tumour behaviour, ${ }^{16}$ whereas in other series their independent loss has been considered to be less important. ${ }^{14}$ As men-

Table 5 Independent predictors of overall survival in Cox proportional hazards model

\begin{tabular}{lll}
\hline Variable & Hazards ratio (95\% CI) & Significance (p value) \\
\hline T class & 1.00 & 0.0003 \\
T1 & $1.25(0.48$ to 3.26$)$ & - \\
T2 & $2.56(0.91$ to 7.17$)$ & 0.65 \\
T3 & $4.46(1.78$ to 11.18$)$ & 0.07 \\
T4 & $0.45(0.26$ to 0.77$)$ & 0.001 \\
Performance index (Karnofsky) & $1.83(1.01$ to 3.31$)$ & 0.004 \\
Nuclear $\beta$ catenin expression & & 0.04 \\
\hline
\end{tabular}

CI, confidence interval. tioned earlier, the combination of $\alpha$ catenin with $\beta$ or $\gamma$ catenin is required for normal $\mathrm{E}$ cadherin mediated adhesion. The reduced expression of a single catenin can thus effect the function of the whole cadherin-catenin complex. Although $\alpha$ catenin is crucial for this function, only $\beta$ and $\gamma$ catenins can ensure normal and fully functional $\mathrm{E}$ cadherin mediated adhesion. $^{34}$

In our study, the expression of at least one catenin was reduced in $69(60 \%)$ tumours, and the expression patterns of all catenins showed a significant association with each other. In previous studies on head and neck cancer, the coordinated expression of $\alpha, \beta$, or $\gamma$ catenins has either not been investigated, ${ }^{15}{ }^{19} 20$ or has not been detected, ${ }^{14}{ }^{16}$ and in breast carcinoma a significant association between $\beta$ and $\gamma$ catenins has been found. ${ }^{24}$ In our series, reduced $\gamma$ catenin expression was significantly related to poor differentiation, but no association was seen between $\alpha$ or $\beta$ catenin expression and clinicopathological characteristics. In previous studies on the head and neck region, reduced expression of catenins was reported to be associated with dedifferentiation of the tumour, ${ }^{14} 151719$ whereas in the reports by Lou and colleagues ${ }^{20}$ and Bagutti et $a l,{ }^{16}$ no such association was noted. Reduced expression of $\alpha$ catenin $^{17}$ as well as $\beta$ catenin ${ }^{18}$ has been noted in clinically more advanced tumours. On the other hand, in other studies on head and neck carcinoma, advanced stage was not associated with catenin expression. ${ }^{14} 151920$ In the oesophageal dysplasia-adenocarcinoma sequence, only $\beta$ catenin expression is reduced with disease progression, whereas $\alpha$ and $\gamma$ catenin expression remains normal. ${ }^{31}$ In bladder carcinoma, reduced $\alpha, \beta$, and $\gamma$ catenin expression is associated with higher histological grade, and reduced $\alpha$ and $\gamma$ catenin expression with advanced tumour stage. ${ }^{26}$ As can be noted from these results, the role of the various catenins in cancer development and progression is unclear. In the head and neck region this might be partly because of the small number of investigations, the small number of samples studied, combining different regions with differently behaving tumours into the same study, and differences in recording and analysing catenin expression. In addition, the differences in specimen preparation procedures, as suggested by Bagutti et al, ${ }^{16}$ can cause discrepancies.

Nuclear $\beta$ catenin expression was positive in $23 \%$ of the tumours in our cohort. Previously, $\beta$ and $\gamma$ catenins have been demonstrated in the nucleus under certain conditions. ${ }^{32}{ }^{33}$ The aberrant localisation of $\beta$ catenin in the cell nucleus has been found in many tumours. ${ }^{25}{ }^{34}$ ${ }_{42}$ In our series, the patients with nuclear $\beta$ catenin expression in their tumours had lower overall survival in the univariate analysis than those patients with no nuclear expression, which is a new and interesting finding in SCCHN. In addition, nuclear $\beta$ catenin expression, as well as tumour status ( $\mathrm{T}$ class) and Karnofsky's performance status were independent prognostic factors of overall survival in multivariate analysis. Nuclear 
expression of $\beta$ catenin has previously been detected in the head and neck region only in nasopharyngeal carcinoma, but without an association with survival. ${ }^{18}$ In line with our observation, an association between nuclear $\beta$ catenin expression and short survival has been reported in colorectal and hepatocellular carcinoma. ${ }^{35} 38$

The actions of catenins in signalling pathways have only been partially clarified. The most investigated pathway associated with catenins in humans is the so called wnt signalling pathway $^{43}$ (named from the combination of the names of homologue pathways in other species: "wingless" (Wg) in drosophila and "int-1" in the mouse (Wnt-1)). ${ }^{53}$ Without wnt signalling, $\beta$ catenin is readily phosphorylated and eventually degraded. ${ }^{5344}$ The activation of the wnt signalling pathway may block the turnover of uncomplexed $\beta$ catenin, resulting in increased cytoplasmic accumulation. $\beta$ Catenin may then associate with transcription factors of the $\mathrm{T}$ cell transcription factor (TCF)/lymphoid enhancer binding factor 1 (LEF-1) family, translocate to the nucleus, and alter the transcription of target genes. ${ }^{33} 4344$ In cancer, pathological stabilisation of $\beta$ catenin, its association with TCF/LEF-1 transcription factors, nuclear translocation, and oncogenic activation might result from mutations of various wnt signalling pathway components. ${ }^{44}$ In addition, overexpression of the integrin linked kinase induces stabilisation of $\beta$ catenin and further activates LEF- $1 / \beta$ catenin dependent transcription, which indicates an association between wnt and integrin signalling pathways and oncogenic transformation. ${ }^{43}$ Components of the wnt signalling pathway have also been implicated in human tumours ${ }^{753742}$ and in experimental cancer models. ${ }^{32}{ }^{41}$ Recently, mutations of $\beta$ catenin and/or APC (adenomatous polyposis coli) have been reported in colon, ${ }^{7}$ anaplastic thyroid, ${ }^{25}$ and hepatocellular cancer, ${ }^{37}{ }^{38}$ as well as in malignant melanoma. ${ }^{42}$ In all but one of these reports, mutations were accompanied by nuclear localisation of $\beta$ catenin, ${ }^{25} 373842$ suggesting that similar mechanisms might be responsible for nuclear $\beta$ catenin expression and the shortened survival detected in our study.

Archival material has previously been used successfully for the immunohistochemical investigation of catenin expression. ${ }^{815}{ }^{17}$ In line with earlier papers, ${ }^{9} 1018$ heterogeneous expression of all catenins was evident in many tumours, probably because of varying intratumoral differentiation. We think that our results are reliable because normal epithelial and glandular tissue were used as internal positive controls in each section, and external positive and negative controls were included in each staining batch. The present evaluation method has been used previously, ${ }^{9}{ }^{10} 2631$ and seems to be reliable for scoring purposes.

Although information on the function of catenins in cell adhesion, as well as in signalling, is increasing fast the importance of their expression in PSCC is unclear. New data on their expression and regulation in tumour cell biology will undoubtedly advance our knowledge of tumour cell behaviour, as has been emphasised by Bagutti et al. ${ }^{16}$ Here, we demonstrate in a large series of head and neck carcinomas of oropharyngeal and hypopharyngeal origin, that reduced expression of $\gamma$ catenin is associated with dedifferentiation of the tumour. Zheng et al have previously reported nuclear $\beta$ catenin expression in head and neck cancer, ${ }^{18}$ but did not comment on its biological relevance. Interestingly, nuclear $\beta$ catenin expression detected in our series independently predicted a shortened overall survival. This is the first report of the biological importance of nuclear $\beta$ catenin in cancer of the head and neck. Our results indicate that nuclear $\beta$ catenin should be investigated in carcinomas of this region, especially because they suggest that nuclear $\beta$ catenin expression could be a valuable prognostic marker in PSCC.

This study was financially supported by the Northern Savo Cancer Fund and Kuopio University Hospital EVO funds. The authors thank Mrs A Parkkinen for the skilful immunohistochemical assistance and Mrs P Halonen for assistance with stachemical assistance
tistical analysis.

1 Berrino F, Gatta G. Variation in survival of patients with head and neck cancer in Europe by the site of origin of the tumours. Eur f Cancer 1998;34:2154-61.

2 Ozawa M, Baribault H, Kemler R. The cytoplasmic domain of the cell adhesion molecule uvomorulin associates with three independent proteins structurally related in different species. EMBO f 1989;8:1711-17.

3 Aberle H, Schwartz H, Kemler R. Cadherin-catenin complex: protein interactions and their implications for cadherin function. 7 Cell Biochem 1996;61:514-23.

4 Cowin P, Burke B. Cytoskeleton-membrane interactions. Curr Opin Cell Biol 1996;8:56-65.

5 Miller JR, Moon RT. Signal transduction through $\beta$-catenin Miller JR, Moon RT. Signal transduction through $\beta$-catenin
and specification of cell fate during embryogenesis. Genes and specification of cell

6 Hoschuetzky H, Aberle H, Kemler R. $\beta$-Catenin mediates the interaction of the cadherin-catenin complex with epidermal growth factor receptor. F Cell Biol 1994;127: $1375-80$.

7 Morin PJ, Sparks AB, Korinek V, et al. Activation of $\beta$-catenin-Tcf signaling in colon cancer by mutations in B-catenin or APC. Science 1997;275:1787-90.

8 Jawhari A, Jordan S, Poole S, et al. Abnormal immunoreactivity of the E-cadherin-catenin complex in gastric carcinoma: relationship with patient survival. Gastroenterology 1997;112:46-54.

9 Nakanishi Y, Ochiai A, Akimoto S, et al. Expression of E-cadherin, $\alpha$-catenin, $\beta$-catenin and plakoglobin in esophageal carcinomas and its prognostic significance: immunohistochemical analysis of 96 lesions. Oncology immunohistochemica

10 Ropponen KM, Eskelinen MJ, Lipponen PK, et al. Reduced expression of $\alpha$ catenin is associated with poor prognosis in colorectal carcinoma. F Clin Pathol 1999;52:10-16.

11 Anttila M, Kosma VM, Ji H, et al. Clinical significance of $\alpha$-catenin, collagen IV, and Ki-67 expression in epithelial ovarian cancer. F Clin Oncol 1998;16:2591-600.

12 Aaltomaa S, Lipponen P, Ala-Opas M, et al. $\alpha$-Catenin expression has prognostic value in local and locally advanced prostate cancer. Br f Cancer 1999;80:477-82.

13 Retera JM, Leers MP, Sulzer MA, et al. The expression of $\beta$-catenin in non-small-cell lung cancer: a clinicopathological study. F Clin Pathol 1998;51:891-4.

14 Andrews NA, Jones AS, Helliwell TR, et al. Expression of the E-cadherin-catenin cell adhesion complex in primary squamous cell carcinomas of the head and neck and their squamous cell carcinomas of the head and neck
nodal metastases. Br f Cancer 1997;75:1474-80.

15 Williams HK, Sanders DS, Jankowski JA, et al. Expression of cadherins and catenins in oral epithelial dysplasia and squamous cell carcinoma. F Oral Pathol Med 1998;27:30817

16 Bagutti C, Speight PM, Watt FM. Comparison of integrin, cadherin, and catenin expression in squamous cell carcinomas of the oral cavity. I Pathol 1998;186:8-16.

17 Hirvikoski P, Kumpulainen EJ, Virtaniemi JA, et al. Cytoplasmic accumulation of $\alpha$-catenin is associated with aggressive features in laryngeal squamous-cell carcinoma. Int $\mathcal{A}$ Cancer 1998;79:546-50.

18 Zheng Z, Pan J, Chu B, et al. Downregulation and abnormal expression of E-cadherin and $\beta$-catenin in nasopharyngeal carcinoma: close association with advanced disease stage and lymph node metastasis. Hum Pathol 1999;30:458-66.

19 Lo Muzio L, Staibano S, Pannone G, et al. Beta- and gamma-catenin expression in oral squamous cell carcinomas. Anticancer Res 1999;19:3817-26. 
20 Lou P, Chen W, Sheen T, et al. Expression of E-cadherin/ catenin complex in nasopharyngeal carcinoma: correlation with clinicopathological parameters. Oncol Rep 1999;6: 1065-71.

21 UICC. In: Sobin LH, Wittekind C, eds. TNM classification of malignant tumours, 5th ed. New York: Wiley-Liss, 1997:25-32.

22 Karnofsky DA, Abelmann WH, Craver LF, et al. The use of nitrogen mustards in the palliative treatment of carcinoma. Cancer 1948;1:634-56.

23 Shanmugaratnam K, Sobin LH. Histological typing of tumours of the upper respiratory tract and ear, 2nd ed. Heidelberg: Springer-Verlag, 1991

24 Bukholm IK, Nesland JM, Kåresen R, et al. E-cadherin and $\alpha-, \beta-$, and $\gamma$-catenin protein expression in relation to metastasis in human breast carcinoma. 7 Pathol $1998 \cdot 185$. 262-6.

25 Garcia-Rostan G, Tallini G, Herrero A, et al. Frequent mutation and nuclear localization of $\beta$-catenin in anaplastic mutation and nuclear localization of $\beta$-catenin in

26 Syrigos KN, Harrington K, Waxman J, et al. Altered $\gamma$-catenin expression correlates with poor survival in $\gamma$-catenin expression correlates with poor survival
patients with bladder cancer. F Urol 1998;160:1889-93.

27 Schipper JH, Unger A, Jahnke K. E-cadherin as a functional marker of the differentiation and invasiveness of squamous cell carcinoma of the head and neck. Clin Otolaryngol 1994; 19:381-4.

28 Mattijssen V, Peters HM, Schalkwijk L, et al. E-Cadherin expression in head and neck squamous-cell carcinoma is associated with clinical outcome. Int f Cancer 1993;55: $580-5$.

29 Bowie GL, Caslin AW, Roland NJ, et al. Expression of the cell-cell adhesion molecule E-cadherin in squamous cell carcinoma of the head and neck. Clin Otolaryngol 1993;18: 196-201.

30 Schipper JH, Frixen UH, Behrens J, et al. E-cadherin expression in squamous cell carcinomas of head and neck: inverse correlation with tumor dedifferentiation and lymph node metastasis. Cancer Res 1991;51:6328-37.

31 Bailey T, Biddlestone L, Shepherd N, et al. Altered cadherin and catenin complexes in the Barrett's esophagusdysplasia-adenocarcinoma sequence: correlation with disdysplasia-adenocarcinoma sequence: correlation with disease progression
32 Simcha I, Shtutman M, Salomon D, et al. Differential nuclear translocation and transactivation potential of

33 Behrens J, von Kries JP, Kühl $M$, et al. Functional interaction of $\beta$-catenin with the transcription factor LEF-1. Nature 1996;382:638-42.

34 Back W, Loff S, Jenne D, et al. Immunolocalisation of beta catenin in intestinal polyps of Peutz-Jeghers and juvenile polyposis syndromes. F Clin Pathol 1999;52:345-9.

35 Hugh TJ, Dillon SA, Taylor BA, et al. Cadherin-catenin expression in primary colorectal cancer: a survival analysis. Brf Cancer 1999;80:1046-51.

36 Hao X, Tomlinson I, Ilyas $\mathrm{M}$, et al. Reciprocity between membranous and nuclear expression of $\beta$-catenin in colorectal tumours. Virchows Arch 1997;431:167-72.

37 de La Coste A, Romagnolo B, Billuart P, et al. Somatic mutations of $\beta$-catenin gene are frequent in mouse and human hepatocellular carcinomas. Proc Natl Acad Sci USA 1998;95:8847-51.

38 Nhieu JT, Renard CA, Wei Y, et al. Nuclear accumulation of mutated $\beta$-catenin in hepatocellular carcinoma is associated with increased cell proliferation. Am 7 Pathol 1999;155:703-10.

39 Fukuchi $T$, Sakamoto $M$, Tsuda $H$, et al. $\beta$-Catenin mutation in carcinoma of the uterine endometrium. Cancer Res 1998;58:3526-8.

40 Kimura Y, Shiozaki H, Doki Y, et al. Cytoplasmic $\beta$-catenin in esophageal cancers. Int $\mathcal{F}$ Cancer 1999;84:174-8.

41 Sormunen RT, Leong AS, Vääräniemi JP, et al. Immunolocalization of the fodrin, E-cadherin, and $\beta$-catenin adhesion complex in infiltrating ductal carcinoma of the breastcomparison with an in vitro model. F Pathol 1999;187:41623.

42 Rimm DL, Caca K, Hu G, et al. Frequent nuclear/ cytoplasmic localization of $\beta$-catenin without exon 3 mutations in malignant melanoma. Am f Pathol 1999;154:3259.

43 Pötter E, Bergwitz C, Brabant G. The cadherin-catenin system: implications for growth and differentiation of system: implications for growth and differe

44 Behrens J. Cadherins and catenins: role in signal transduction and tumor progression. Cancer Metastasis Rev 1999;18:15-30. 